Check for updates

Cite this: J. Mater. Chem. A, 2021, 9 , 19719

Received 2nd April 2021

Accepted 17th May 2021

DOI: $10.1039 / d 1 t a 02783 b$

rsc.li/materials-a

\section{A crystalline/amorphous CoP@CoB hierarchical core-shell nanorod array for enhanced hydrogen evolution $\uparrow$}

\author{
Peidong Shi, $\grave{\dagger}^{\mathrm{a}}$ Yu Zhang, $\dot{\dagger}^{\mathrm{a}}$ Guanglu Zhang, ${ }^{\mathrm{b}}$ Xiaojuan Zhu, ${ }^{\mathrm{a}}$ Shaohua Wang ${ }^{\mathrm{a}}$ \\ and An-Liang Wang (D)*a
}

\begin{abstract}
Highly active, durable and cost-effective catalysts toward the hydrogen evolution reaction (HER) are crucial for widespread use of electrochemical water splitting in hydrogen production. Herein, a hierarchical coreshell nanorod array (NRA) comprising an inner crystalline CoP nanorod and an outer amorphous CoB (a$\mathrm{CoB}$ ) nanosheet (CoP(a-CoB) is fabricated on conductive carbon cloth through a successive phosphidation-chronoamperometry-boronation strategy. The as-obtained catalyst exhibits excellent HER activity with low overpotentials of 56.3 and $81.2 \mathrm{mV}$ at a current density of $10 \mathrm{~mA} \mathrm{~cm} \mathrm{~cm}^{-2}$ in alkaline and acidic electrolytes, respectively. Furthermore, it exhibits superior long-term stability with almost no activity degradation. The outstanding HER electrocatalytic performance might be attributed to the unique hierarchical core-shell NRA structure with the crystalline CoP nanorod as the core and the amorphous $\mathrm{COB}$ nanosheet as the shell, which could not only provide abundant active sites, but also guarantee effective electron transport. This work opens up a promising way to rationally design highefficient electrocatalysts for hydrogen production.
\end{abstract}

Hydrogen is widely considered as one promising alternative to traditional fossil fuels due to its high gravimetric energy density and pollution-free characteristics. ${ }^{1-3}$ Electrochemical water splitting by using electricity from the intermittent renewable solar energy is an economic, sustainable and environmentfriendly approach for green hydrogen production. ${ }^{4-7}$ Currently, Pt based electrocatalysts are still the most active ones for the hydrogen evolution reaction (HER).${ }^{8-10}$ However, their widespread commercial applications are greatly restricted by their low abundance on the earth, high cost, and unsatisfactory stability. Therefore, it is of great significance to develop earthabundant and cost-effective catalysts with high activity and excellent stability for the hydrogen economy.

Recently, Co based compounds have been explored to be attractive candidates to replace Pt based catalysts, due to their earth-abundance, low cost and ease of scale-up, such as CoO, $\mathrm{CoP}, \mathrm{CoSe}_{2}$, and $\mathrm{CoS}_{2} \cdot{ }^{11-17}$ Nevertheless, the HER performances of

\footnotetext{
${ }^{a}$ Key Laboratory for Colloid and Interface Chemistry Ministry of Education, School of Chemistry and Chemical Engineering, Shandong University, Jinan 250100, Shandong, China.E-mail: alwang@sdu.edu.cn

${ }^{b}$ College of Chemistry, Chemical Engineering and Materials Science, Collaborative Innovation Center of Functionalized Probes for Chemical Imaging in Universities of Shandong, Key Laboratory of Molecular and Nano Probes, Ministry of Education, Institute of Biomedical Sciences, Shandong Normal University, Jinan 250014, Shandong, China

$\dagger$ Electronic supplementary information (ESI) available: Experimental section and supplementary figures. See DOI: $10.1039 / \mathrm{d} 1$ ta02783b

\$ These authors contributed equally to this work.
}

those Co based compounds still need to be enhanced to reach the level of Pt-based catalysts. It has been demonstrated that rationally tailoring the crystallinity and nanostructure of an electrocatalyst is considered as one highly efficient approach to improving its intrinsic activity. ${ }^{18-20}$ Compared with crystalline materials, amorphous counterparts with a disordered atomic arrangement could normally provide abundant active sites, plenty of defects and unsaturated coordination sites, which could greatly boost the electrocatalytic activity. ${ }^{21-23}$ However, generally, amorphous materials possess low electronic conductivity, causing an extra energy barrier and thus leading to performance degradation..$^{24,25}$ One promising solution is to construct the coreshell nanoarray structure, especially with a conductive core and amorphous shell, which can not only facilitate rapid charge transfer, but also increase the number of exposed active sites. ${ }^{26-28}$ Moreover, the strong interaction between the core and the shell can modulate the electronic structure of active sites, which could be beneficial for the activity improvement. ${ }^{29,30}$ More importantly, the self-supported nanoarray on conductive substrates (i.e. carbon cloth, carbon paper) without usage of binders (i.e. Nafion) could accelerate mass diffusion, expedite release of accumulated gas bubbles, and prevent the catalyst from delamination. ${ }^{31-34}$ Therefore, it is desirable to design and synthesize a Co-based core-shell nanoarray with conductive core and amorphous shell to improve the HER performance.

Based on the above considerations, a novel, hierarchical core-shell nanorod array (NRA) was integrated on carbon cloth 
(CC), in which the amorphous CoB (a-CoB) nanosheet (NS) is in situ grown on the surface of the crystalline CoP NRA. During the fabrication, the CoP NRA on CC was transformed into a $\mathrm{CoP} @ \mathrm{Co}(\mathrm{OH})_{2}$ hierarchical NRA (HNRA) by chronoamperometry electrolysis, which was subsequently converted into CoP@a-CoB HNRA via boronizing treatment. It is found that the hierarchical and crystalline-amorphous core-shell structure can not only greatly increase the amount of exposed active sites, but also expedite the electron and mass transfer. The as-synthesized CoP@a-CoB HNRA exhibits excellent HER activity with low overpotentials of 56.3 and $81.2 \mathrm{mV}$ to deliver the current density of $10 \mathrm{~mA} \mathrm{~cm}{ }^{-2}$ in alkaline and acidic solutions, and is much better than $\mathrm{CoP}$, a-CoB and most reported Co based HER electrocatalysts. Moreover, the CoP@a-CoB HNRA displays excellent durability with almost no potential change during long-term electrolysis. This work provides an effective strategy to synthesize efficient and stable electrocatalysts for the HER.

The fabrication procedure of the CoP@a-CoB HNRA on CC is illustrated in Fig. 1. The detailed process is presented in the ESI. $\dagger$ Firstly, the $\mathrm{Co}\left(\mathrm{CO}_{3}\right)_{0.5}(\mathrm{OH})_{0.11} \cdot \mathrm{H}_{2} \mathrm{O}(\mathrm{CoCH})$ NRA was firstly synthesized on CC by a low temperature hydrothermal method. Scanning electron microscopy (SEM) images and the X-ray diffraction (XRD) pattern demonstrate the successful synthesis of the CoCH NRA (Fig. S1 and S2 $\dagger$ ). Afterward, the CoCH NRA was phosphatized using $\mathrm{Na}_{2} \mathrm{H}_{2} \mathrm{PO}_{2}$ at $300{ }^{\circ} \mathrm{C}$ for $2 \mathrm{~h}$ to obtain the CoP NRA. The XRD pattern confirms the formation of CoP (Fig. S3†). As indicated by the SEM and transmission electron microscopy (TEM) images of the CoP NRA, the NRA structure is well maintained after phosphorization (Fig. 2a and b). Subsequently, the $\mathrm{CoP} @ \mathrm{Co}(\mathrm{OH})_{2}$ HNRA was achieved by chronoamperometry electrolysis at $-0.16 \mathrm{~V}$ (vs. the reversible hydrogen electrode, RHE) for $8 \mathrm{~h}$ by using CoP NRA/CC as the working electrode. In the XRD pattern of the $\mathrm{CoP} @ \mathrm{Co}(\mathrm{OH})_{2}$ HNRA (Fig. S4 $\dagger$ ), the formation of $\mathrm{Co}(\mathrm{OH})_{2}$ is confirmed by the appearance of some new diffraction peaks, located at $19.1^{\circ}$, $32.5^{\circ}, 38.0^{\circ}, 51.4^{\circ}, 57.9^{\circ}, 59.6^{\circ}, 61.5^{\circ}, 69.5^{\circ}$ and $71.3^{\circ}$, which can be indexed to the (001), (100), (101), (102), (110), (003), (111), (103), and (201) planes of $\mathrm{Co}(\mathrm{OH})_{2}$, respectively. The SEM and TEM images show that $\mathrm{CoP}\left(\mathrm{Co}(\mathrm{OH})_{2}\right.$ displays a hierarchical structure, in which the surface of the nanorod is covered with plenty of ultrathin nanosheets (Fig. S5 $\dagger$ ). As shown in the highresolution TEM (HRTEM) image of the CoP@Co(OH $)_{2}$ HNRA,

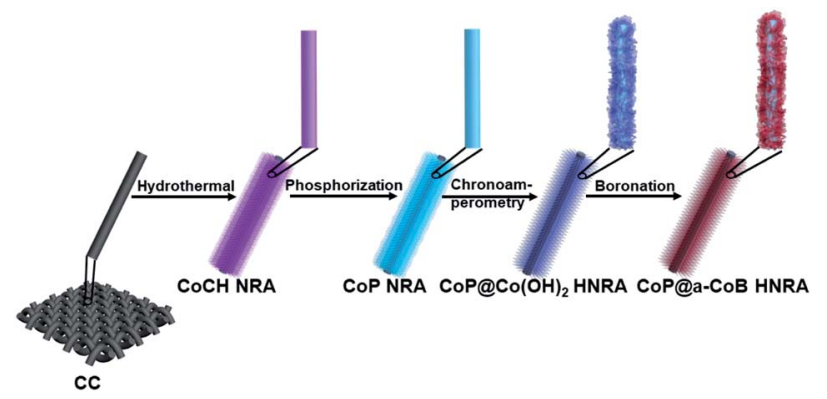

Fig. 1 Schematic illustration of the synthesis process of the CoP@aCOB HNRA on CC.

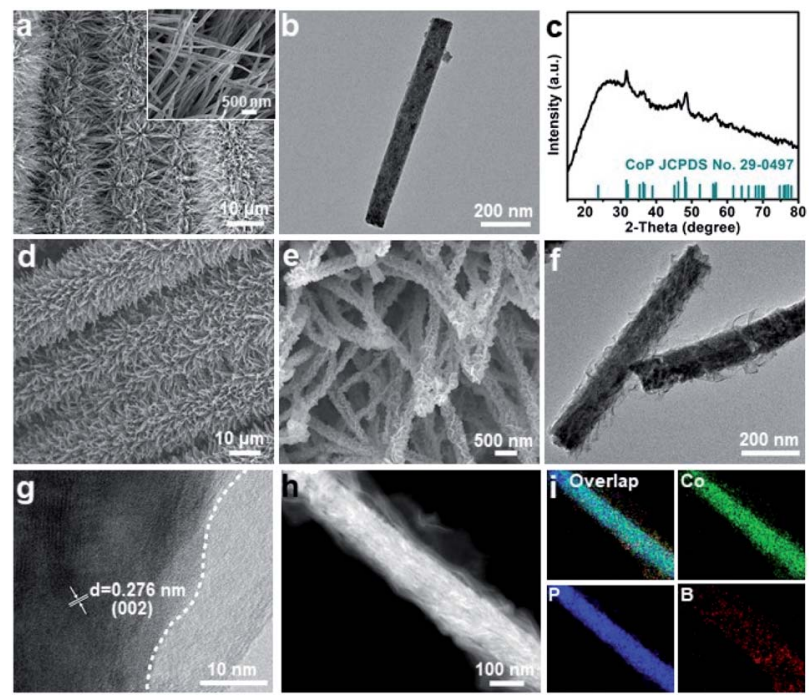

Fig. 2 (a) SEM image (inset is the enlarged SEM image) of the COP NRA on CC. (b) TEM image of one typical CoP nanorod. (c) XRD pattern of the CoP@a-CoB HNRA. SEM images of the CoPaa-CoB HNRA on CC with (d) low and (e) high magnification. (f) TEM image of two CoP@aCoB hierarchical nanorods. (g) HRTEM image of the CoP@a-CoB hierarchical nanorod. (h) HAADF-STEM image of one typical CoP(aCoB hierarchical nanorod. (i) The corresponding EDS elemental mapping images.

the lattice distances of 3.74 and $2.78 \AA$ from the inner nanorod and outer nanosheet correspond to the (101) plane of CoP and the (100) plane of $\mathrm{Co}(\mathrm{OH})_{2}$, respectively, which confirms the structure of the $\mathrm{CoP} @ \mathrm{Co}(\mathrm{OH})_{2}$ HNRA. After boronizing treatment in $\mathrm{NaBH}_{4}$ solution at room temperature, the final CoP@aCoB HNRA was obtained on CC. For comparison, the a-CoB NS was also prepared using a modified reported method. ${ }^{35}$ The TEM image and XRD pattern are shown in Fig. S7 and S8. $\dagger$

The XRD pattern of the CoP@a-CoB HNRA only shows the diffraction peaks belonging to CoP, and the absence of $\mathrm{CoB}$ diffraction peaks suggests the amorphous nature of $\mathrm{CoB}$ (Fig. 2c). The SEM images clearly display the hierarchical nanorods, which are uniformly and perpendicularly integrated onto the surface of CC and separated from each other (Fig. 2d and e). The TEM image of two typical CoP@a-CoB hierarchical nanorods indicates that the surface of the nanorod is covered with plenty of ultrathin nanosheets (Fig. 2f). The CoP@a-CoB hierarchical nanorod is further demonstrated by HRTEM, and the resolved lattice fringes with an interplanar distance of 2.67 A located in the inner nanorod correspond to the (002) plane of CoP, whereas no lattice fringe in the region of the outer nanosheet suggests the amorphous nature of $\mathrm{CoB}$ (Fig. 2g). Fig. 2h illustrates the high-angle annular dark-field scanning TEM (HAADF-STEM) image of one CoP@a-CoB hierarchical nanorod, and the corresponding energy-dispersive X-ray spectroscopy (EDS) mapping (Fig. 2i) and line-scan profile (Fig. S9†) indicate the existence of the inner CoP nanorod and outer a-CoB NS. This unique HNRA structure contains a 1D nanorod, 2D nanosheet, and 3D nanoarray, which will provide a large amount of active sites and efficient electron transport, thus boosting the electrocatalytic activity. Moreover, modulating and constructing 
the interface between the crystalline core and amorphous shell have been proven to be an efficient strategy to enhance the catalytic performance. ${ }^{36,37}$ In this regard, it is expected that the existence of abundant $\mathrm{CoP} / \mathrm{a}-\mathrm{CoB}$ interfaces can endow the CoP@a-CoB HNRA with outstanding HER activity because of the facilitated electron transport and modified electron structure on the $\mathrm{CoP} / \mathrm{a}-\mathrm{CoB}$ interface.

In order to investigate the compositions, chemical states and electronic interactions of the as-synthesized samples, X-ray photoelectron spectroscopy (XPS) measurements were performed on the CoP NRA and CoP@a-CoB HNRA. The existence of Co, B, P in CoP@a-CoB HNRA is confirmed by the XPS full spectrum, while that of the CoP NRA shows the absence of $B$ (Fig. 3a). Fig. 3b is the high-resolution Co $2 \mathrm{p}$ spectrum of the CoP@a-CoB HNRA, which displays four pair of peaks arising from the spin-orbit doublet of Co 2p, corresponding to the Co$\mathrm{B}, \mathrm{Co}-\mathrm{P}, \mathrm{Co}-\mathrm{O}$ and satellites. The appearance of oxide species might be due to the surface oxidation of CoP@a-CoB exposed in air. It is noted that the binding energies of Co $2 \mathrm{p}_{3 / 2}$ and $2 \mathrm{p}_{1 / 2}$ of the Co-P bond in the CoP@a-CoB HNRA (778.2 eV and 793.2 eV) shift to lower values ( $c a .0 .7 \mathrm{eV}$ ) compared with those in the CoP NRA (778.9 eV and 793.9 eV). In the high-resolution B 1s spectrum of the CoP@a-CoB HNRA, there are two peaks located at $186.9 \mathrm{eV}$ and $191.7 \mathrm{eV}$, corresponding to the Co-B and oxidized $\mathrm{B}$, respectively (Fig. 3c). In the $\mathrm{P} 2 \mathrm{p}$ spectrum of the $\mathrm{CoP} @ \mathrm{a}-\mathrm{CoB}$ HNRA, two peaks located at $128.5 \mathrm{eV}$ and $129.5 \mathrm{eV}$ can be observed, which are assigned to the $\mathrm{P} 2 \mathrm{p}_{3 / 2}$ and $2 \mathrm{p}_{1 / 2}$ of Co-P, respectively, along with a peak at $132.7 \mathrm{eV}$ originating from the oxidized phosphorous species (Fig. 3d). Obviously, the CoP@aCoB HNRA exhibits a weak $\mathrm{P} 2 \mathrm{p}$ signal intensity and a large ratio of the Co-P bond in P 2p, compared with the CoP NRA, which may be due to the distribution of the a-CoB NS on the surface of $\mathrm{CoP}$, protecting CoP from being oxidized. More importantly, the
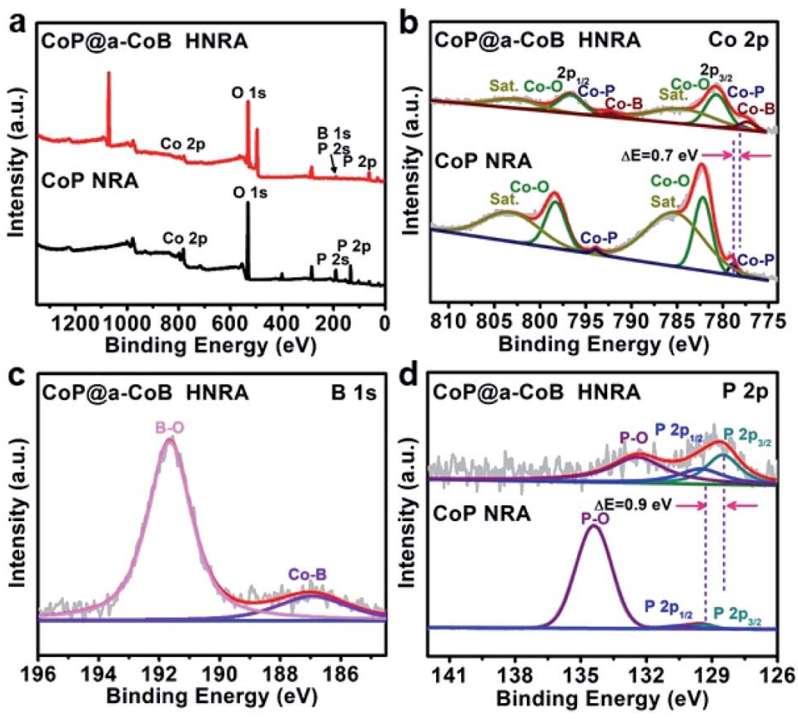

Fig. 3 (a) Full XPS spectra of the CoP NRA and CoP(aa-CoB HNRA. (b) High-resolution Co $2 p$ XPS spectra of the CoP NRA and CoP@a-CoB HNRA. (c) High-resolution B 1s XPS spectrum of the CoPaa-CoB HNRA. (d) High-resolution P $2 p$ XPS spectra of the CoP NRA and CoP@a-COB HNRA.
P $2 \mathrm{p}_{3 / 2}$ and $2 \mathrm{p}_{1 / 2}$ peaks of the CoP@a-CoB HNRA both shift to lower binding energy ( $c a .0 .9 \mathrm{eV}$ ) compared with those of the CoP NRA. Therefore, the apparently negative shifts of both Co $2 \mathrm{p}$ and $\mathrm{P} 2 \mathrm{p}$ in the CoP@a-CoB HNRA suggest that there exists strong electronic interaction between $\mathrm{CoP}$ and a-CoB, which could modulate the electronic structure of the active sites, thus promoting the HER electrocatalytic kinetics. ${ }^{38}$

The electrocatalytic activity of the CoP@a-CoB HNRA toward the HER was evaluated by linear sweep voltammetry (LSV) with a scan rate of $10 \mathrm{mV} \mathrm{s}^{-1}$ in $\mathrm{H}_{2}$-saturated 1.0 $\mathrm{M} \mathrm{KOH}$. Herein, the CoP@a-CoB HNRA supported on CC was directly used as the working electrode. For comparison, the CC, CoP NRA, a-CoB NS, and commercial $\mathrm{Pt} / \mathrm{C}$ catalysts were tested under the same conditions. All experimental data were corrected with ohmic potential drop $(i R)$ losses for further analysis. From the polarization curves (Fig. 4a), it can be seen that the commercial Pt/C is highly active for the HER with an onset potential of almost $0 \mathrm{mV}$, whereas the CC substrate and a-CoB NS show very poor HER activity. Note that the CoP@a-CoB HNRA displays excellent catalytic activity with an overpotential of $56.3 \mathrm{mV}$ to deliver the current density of $10 \mathrm{~mA} \mathrm{~cm}{ }^{-2}$, much better than those of CoP NRA $(80.3 \mathrm{mV})$ and a-CoB NS $(338.6 \mathrm{mV})$. At the overpotential of $120 \mathrm{mV}$, the CoP@a-CoB HNRA exhibits a much higher current density than the CoP NRA (Fig. 4b). More importantly, when the overpotential exceeds $64.8 \mathrm{mV}$, the HER current density of the
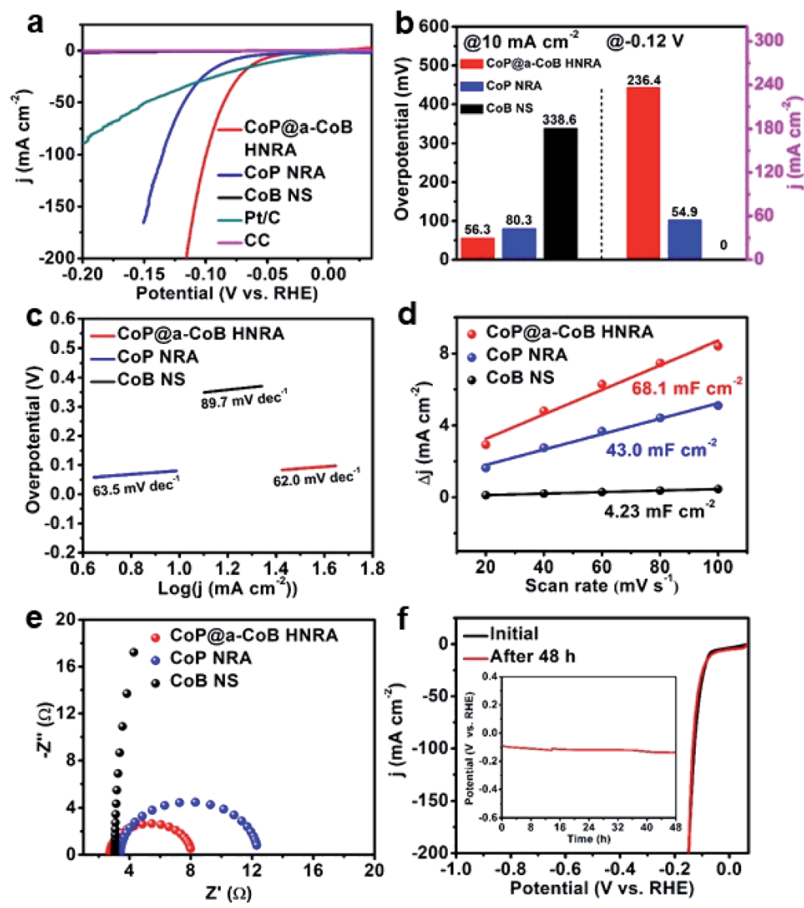

Fig. 4 (a) The polarization curves of different catalysts obtained in $\mathrm{H}_{2}$ saturated $1.0 \mathrm{M} \mathrm{KOH}$ at a scan rate of $10 \mathrm{mV} \mathrm{s}^{-1}$. (b) The histograms of overpotential at $10 \mathrm{~mA} \mathrm{~cm}{ }^{-2}$ and current density at $-0.12 \mathrm{~V} v \mathrm{~s}$. RHE for the CoP@a-CoB HNRA, CoP NRA, and a-CoB NS. (c) The Tafel slopes and (d) plots of capacitive currents as a function of scan rate, (e) Nyquist plots of the CoP@a-CoB HNRA, CoP NRA, and a-CoB NS. (f) The polarization curves of the CoP@a-CoB HNRA before and after the chronopotentiometry test (inset is the chronopotentiometry curve under the current density of $10 \mathrm{~mA} \mathrm{~cm}^{-2}$ ). 
CoP@a-CoB HNRA is even larger than that of commercial Pt/C. The catalytic kinetics of the catalysts were revealed by Tafel plots. The Tafel plots show that the CoP@a-CoB HNRA has a much smaller Tafel slope than the CoP NRA and a-CoB NS, indicating its faster kinetics (Fig. 4c). Therefore, the CoP@aCoB HNRA displays excellent HER activity in alkaline solution with a low overpotential and small Tafel slope, and is better than CoP NRA, a-CoB NS and most reported Co based HER electrocatalysts (Table $\mathrm{S} 1 \dagger$ ).

To gain a deep understanding of the excellent HER activity of the CoP@a-CoB HNRA, electrochemical surface area (ECSA) and electrochemical impedance spectroscopy (EIS) measurements were performed. The electrochemical double layer capacitance $\left(C_{\mathrm{dl}}\right)$, which is used to estimate the ECSA, was calculated from the cyclic voltammetry $(\mathrm{CV})$ curves at different scan rates. The CoP@a-CoB HNRA reveals a $C_{\mathrm{dl}}$ of $68.1 \mathrm{mF} \mathrm{cm}^{-2}$, which is significantly larger than that of the CoP NRA and a-CoB NS, indicating its larger ECSA for HER catalysis (Fig. 4d and S10 $\dagger$ ). The large $C_{\mathrm{dl}}$ of the CoP@a-CoB HNRA certifies that the hierarchical and amorphous structure can create more active sites, and thus improve the HER activity. Meanwhile, the minimum charge-transfer resistance of the CoP@a-CoB HNRA from Nyquist plots suggests a rapid electron transfer process, which could result in boosting HER performance (Fig. 4e). This enhancement in electron transport may be due to the fact that the strong interaction between crystalline CoP and amorphous CoB combined with the self-supported NRA structure reduces the charge-transfer resistance.

To further probe the beneficial aspects of the hierarchical structure of the CoP@a-CoB HNRA, the CoP@a-CoB NRA without hierarchical structure was fabricated by direct boronation of CoP as the reference material (Fig. S11-S13†). As shown in Fig. S14, $\dagger$ the CoP@a-CoB HNRA shows much higher current density than the CoP@a-CoB NRA. Moreover, compared with the CoP@a-CoB HNRA, the CoP@a-CoB NRA possesses a much lower $C_{\mathrm{dl}}\left(25.2 \mathrm{mF} \mathrm{cm}^{-2}\right.$, Fig. S15 $\left.\dagger\right)$, which further confirms that the hierarchical structure is responsible for the excellent HER activity of the CoP@a-CoB HNRA. Therefore, the outstanding HER activity of the CoP@a-CoB HNRAs could be ascribed to the strong interaction between $\mathrm{CoP}$ and a-CoB, the amorphous structure of $\mathrm{CoB}$, the hierarchical (nanosheet on nanorod) structure, and the self-supported NRA structure.

Besides electrocatalytic activity, stability is an another essential quality for an advanced electrocatalyst. The stability of the CoP@a-CoB HNRA was measured by multi-current and chronopotentiometry tests in $1.0 \mathrm{M} \mathrm{KOH}$. Fig. S16† reveals the multi-current stability test carried out at different current densities for the CoP@a-CoB HNRA, and shows that the overpotentials are steady at each current density $(10,20,30,40$, and $50 \mathrm{~mA} \mathrm{~cm}^{-2}$ ). In particular, when the current density is back to $10 \mathrm{~mA} \mathrm{~cm}{ }^{-2}$ after going through a series of high current density flow, the potential resumes and prevails over an additional $2 \mathrm{~h}$. As shown in the chronopotentiometry curve of the CoP@a-CoB HNRA (Fig. 4f), the overpotential at the current density of $10 \mathrm{~mA}$ $\mathrm{cm}^{-2}$ displays a negligible increase for $48 \mathrm{~h}$. Moreover, the polarization curves before and after the $48 \mathrm{~h}$ chronopotentiometry test indicate that there is a negligible decay in
HER activity. These results clearly demonstrate that the CoP@aCoB HNRA possesses excellent electrochemical stability. The CoP@a-CoB HNRA after the chronopotentiometry test was measured by SEM, TEM, XRD and XPS. As shown in Fig. S17, $\dagger$ the self-supported and hierarchical structures of the CoP@aCoB HNRA were perfectly preserved, manifesting outstanding structural stability. Fig. S18 $\dagger$ is the XRD pattern of the CoP@aCoB HNRA after the durability test, in which only peaks belonging to CoP exist, consistent with those in the XRD pattern of the original CoP@a-CoB HNRA. The XPS spectra show no obvious change of chemical states of Co, $\mathrm{P}$ and $\mathrm{B}$, confirming the excellent stability of the CoP@a-CoB HNRA (Fig. S19†).

The development of proton exchange membrane (PEM) water splitting greatly pushes the exploration of HER electrocatalysts under acidic media. Herein, the electrocatalytic HER performance of the CoP@a-CoB HNRA was also investigated in acidic solution. In $0.5 \mathrm{M} \mathrm{H}_{2} \mathrm{SO}_{4}$, the CoP@a-CoB HNRA exhibits excellent HER activity with an overpotential of $81.2 \mathrm{mV}$ at $10 \mathrm{~mA}$ $\mathrm{cm}^{-2}$, which is much lower than that of the CoP NRA $(116.3 \mathrm{mV})$ and a-CoB NS (597.4 mV) (Fig. 5a). Moreover, at the overpotential of $120 \mathrm{mV}$, the CoP@a-CoB HNRA possesses a much higher current density than the CoP NRA and a-CoB NS (Fig. 5b). The Tafel slope of the CoP@a-CoB HNRA is $55.4 \mathrm{mV} \mathrm{dec}-1$ (Fig. 5c). The EIS and $C_{\mathrm{dl}}$ measurements in $0.5 \mathrm{M} \mathrm{H}_{2} \mathrm{SO}_{4}$ suggest that the CoP@a-CoB HNRA possesses rapid electron-transfer kinetics and abundant active sites, which are beneficial for improving the performance (Fig. S20 and S21 $\dagger$ ). In addition, the CoP@a-CoB HNRA exhibits higher HER activity than the CoP@a-CoB NRA (Fig. S22 †). Furthermore, the durability of the CoP@a-CoB HNRA via the chronopotentiometry test at the current density of $10 \mathrm{~mA} \mathrm{~cm}^{-2}$ (Fig. 5d) was investigated, and it was found that the CoP@a-CoB HNRA shows superior durability for $24 \mathrm{~h}$ without any noticeable degradation through
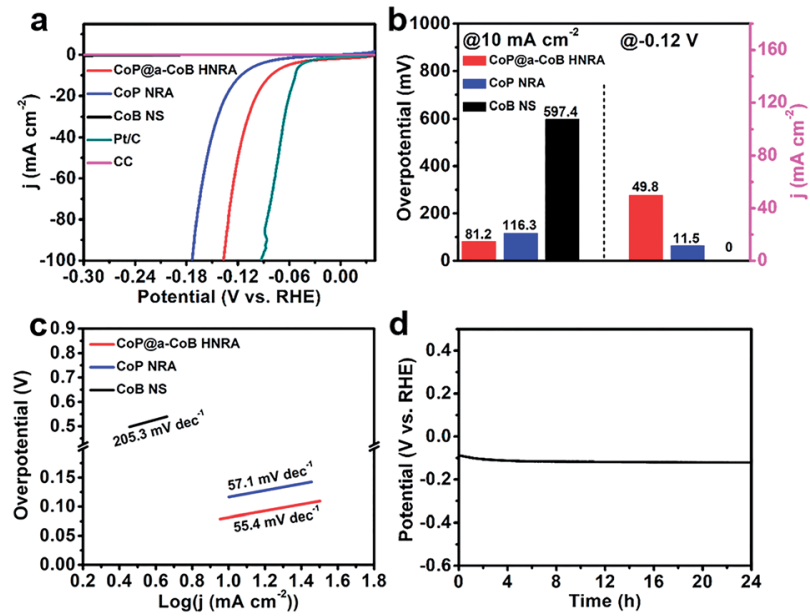

Fig. 5 (a) The polarization curves of different catalysts obtained in $\mathrm{H}_{2}$ saturated $0.5 \mathrm{M} \mathrm{H}_{2} \mathrm{SO}_{4}$ at a scan rate of $10 \mathrm{mV} \mathrm{s}^{-1}$. (b) The histograms of overpotential at $10 \mathrm{~mA} \mathrm{~cm}^{-2}$ and current density at $-0.12 \mathrm{~V} v \mathrm{~s}$. RHE for the CoP(a-CoB HNRA, CoP NRA, and a-CoB NS. (c) The Tafel slopes of the CoPaa-CoB HNRA, CoP NRA, and a-CoB NS. (d) Chronopotentiometry curve of the CoP@a-CoB HNRA at the current density of $10 \mathrm{~mA} \mathrm{~cm}^{-2}$. 
continuous hydrogen generation. Therefore, the CoP@a-CoB HNRA displays excellent HER performance in acidic solution, and is much better than CoP NRA, a-CoB NS and most reported Co based electrocatalysts towards the acidic HER (Table S2 $\dagger$ ).

The superior HER of the CoP@a-CoB HNRA in alkaline and acidic electrolytes can be attributed to the following factors: (1) the amorphous CoB with a disordered atomic arrangement could provide abundant defects and unsaturated coordination sites, boosting the performance. (2) The conductive CoP and self-supported NRA structure solve the limitation of the low conductivity of a-CoB, and guarantees effective electronic transport. (3) The strong interaction between CoP and a-CoB modulates the electronic structure of active sites, which may be beneficial for performance enhancement. (4) The hierarchical structure (nanosheet on nanorod) could provide a large electrochemical surface area and plenty of active sites. (5) The self-supported feature of the CoP@a-CoB HNRA could avoid the usage of a binder, which may block the active sites and decrease the conductivity, and thus will let the CoP@a-CoB HNRA effectively participate in the HER with almost no "dead" volume.

In summary, a CoP@a-CoB hierarchical core-shell NRA supported on CC was designed and prepared via a successive phosphidation-chronoamperometry-boronation strategy. In this HNRA structure, the crystalline CoP nanorod is covered by plenty of amorphous CoB NSs to form the hierarchical coreshell structure, which could not only provide abundant active sites, but also enable effective and fast electron transfer. The asprepared CoP@a-CoB HNRA exhibits excellent electrocatalytic performance toward the HER with overpotentials of 56.3 and $81.2 \mathrm{mV}$ at a current density of $10 \mathrm{~mA} \mathrm{~cm} \mathrm{~cm}^{-2}$ in alkaline and acidic solutions, respectively, and is much better than CoP, a$\mathrm{CoB}$ and most reported Co based HER electrocatalysts. Moreover, the CoP@a-CoB HNRA enables long-term operation with negligible degradation. We believe that this work will open a novel pathway for the development of highly active, non-noble electrocatalysts for hydrogen production.

\section{Conflicts of interest}

There are no conflicts to declare.

\section{Acknowledgements}

This work was supported by the Qilu Young Scholarship Funding of Shandong University. This work was also supported by the National Natural Science Foundation of China (No. 22005176), the Natural Science Foundation of Shandong Province (ZR2020QE014), and the Natural Science Foundation of Jiangsu Province (BK20200228).

\section{References}

1 M. G. Walter, E. L. Warren, J. R. McKone, S. W. Boettcher, Q. Mi, E. A. Santori and N. S. Lewis, Chem. Rev., 2010, 110, 6446-6473.
2 I. Roger, M. A. Shipman and M. D. Symes, Nat. Rev. Chem., 2017, 1, 3.

3 Z.-L. Wang, K. Sun, J. Henzie, X. Hao, C. Li, T. Takei, Y.-M. Kang and Y. Yamauchi, Angew. Chem., Int. Ed., 2018, 57, 5848-5852.

4 Y. Zhang, X. Zhu, G. Zhang, P. Shi and A.-L. Wang, J. Mater. Chem. A, 2021, 9, 5890-5914.

5 L. Li, P. Wang, Q. Shao and X. Huang, Chem. Soc. Rev., 2020, 49, 3072-3106.

6 Y. Jiao, Y. Zheng, M. Jaroniec and S. Z. Qiao, Chem. Soc. Rev., 2015, 44, 2060-2086.

7 Z. Wu, Y. Zhao, H. Wu, Y. Gao, Z. Chen, W. Jin, J. Wang, T. Ma and L. Wang, Adv. Funct. Mater., 2021, 31, 2010437.

8 R. Subbaraman, D. Tripkovic, D. Strmcnik, K.-C. Chang, M. Uchimura, A. P. Paulikas, V. Stamenkovic and N. M. Markovic, Science, 2011, 334, 1256-1260.

9 C. Zhu, A.-L. Wang, W. Xiao, D. Chao, X. Zhang, N. H. Tiep, S. Chen, J. Kang, X. Wang, J. Ding, J. Wang, H. Zhang and H. J. Fan, Adv. Mater., 2018, 30, 1705516.

10 Y. Zheng, Y. Jiao, Y. Zhu, L. H. Li, Y. Han, Y. Chen, A. Du, M. Jaroniec and S. Z. Qiao, Nat. Commun., 2014, 5, 3783.

11 H. Jin, J. Wang, D. Su, Z. Wei, Z. Pang and Y. Wang, J. Am. Chem. Soc., 2015, 137, 2688-2694.

12 K. Li, J. Zhang, R. Wu, Y. Yu and B. Zhang, Adv. Sci., 2016, 3, 1500426.

13 A.-L. Wang, J. Lin, H. Xu, Y.-X. Tong and G.-R. Li, J. Mater. Chem. A, 2016, 4, 16992-16999.

14 J. F. Callejas, C. G. Read, E. J. Popczun, J. M. McEnaney and R. E. Schaak, Chem. Mater., 2015, 27, 3769-3774.

15 C. L. McCarthy, C. A. Downes, E. C. Schueller, K. Abuyen and R. L. Brutchey, ACS Energy Lett., 2016, 1, 607-611.

16 H. Zhang, Y. Li, G. Zhang, T. Xu, P. Wan and X. Sun, J. Mater. Chem. A, 2015, 3, 6306-6310.

17 M. S. Faber, R. Dziedzic, M. A. Lukowski, N. S. Kaiser, Q. Ding and S. Jin, J. Am. Chem. Soc., 2014, 136, 10053-10061.

18 Z. Liang, W. Zhou, S. Gao, R. Zhao, H. Zhang, Y. Tang, J. Cheng, T. Qiu, B. Zhu, C. Qu, W. Guo, Q. Wang and R. Zou, Small, 2020, 16, 1905075.

19 D. Cheng, Z. Wang, C. Chen and K. Zhou, Chem. Mater., 2019, 31, 8026-8034.

20 Z. Dong, F. Lin, Y. Yao and L. Jiao, Adv. Energy Mater., 2019, 9, 1902703.

21 X. Wang, W. Ma, C. Ding, Z. Xu, H. Wang, X. Zong and C. Li, ACS Catal., 2018, 8, 9926-9935.

22 W. Cai, R. Chen, H. Yang, H. B. Tao, H.-Y. Wang, J. Gao, W. Liu, S. Liu, S.-F. Hung and B. Liu, Nano Lett., 2020, 20, 4278-4285.

23 H. Cheng, N. Yang, G. Liu, Y. Ge, J. Huang, Q. Yun, Y. Du, C.-J. Sun, B. Chen, J. Liu and H. Zhang, Adv. Mater., 2020, 32, 1902964.

24 S. Anantharaj and S. Noda, Small, 2020, 16, 1905779.

25 Y. Zhou and H. J. Fan, ACS Mater. Lett., 2021, 3, 136-147.

26 J. Hu, S. Li, Y. Li, J. Wang, Y. Du, Z. Li, X. Han, J. Sun and P. Xu, J. Mater. Chem. A, 2020, 8, 23323-23329.

27 J. Hu, S. Li, Y. Li, J. Wang, Y. Du, Z. Li, X. Han, J. Sun and P. Xu, J. Mater. Chem. A, 2020, 8, 23323-23329. 
28 H. Yoon, H. J. Song, B. Ju and D.-W. Kim, Nano Res., 2020, 13, 2469-2477.

29 S. Das, J. Pérez-Ramírez, J. Gong, N. Dewangan, K. Hidajat, B. C. Gates and S. Kawi, Chem. Soc. Rev., 2020, 49, 2937-3004.

30 Y. Lin, Y. Pan, S. Liu, K. Sun, Y. Cheng, M. Liu, Z. Wang, X. Li and J. Zhang, Appl. Catal., B, 2019, 259, 118039.

31 J. Hou, Y. Wu, B. Zhang, S. Cao, Z. Li and L. Sun, Adv. Funct. Mater., 2019, 29, 1808367.

32 F. Zhang and L. Qi, Adv. Sci., 2016, 3, 1600049.

33 C. Tang, R. Zhang, W. Lu, Z. Wang, D. Liu, S. Hao, G. Du, A. M. Asiri and X. Sun, Angew. Chem., Int. Ed., 2017, 56, 842-846.
34 T. Y. Ma, S. Dai and S. Z. Qiao, Mater. Today, 2016, 19, 265273.

35 X. Wang, J. Liao, H. Li, H. Wang and R. Wang, Int. J. Hydrogen Energy, 2017, 42, 6646-6656.

36 S. Huang, Y. Meng, Y. Cao, F. Yao, Z. He, X. Wang, H. Pan and M. Wu, Appl. Catal., B, 2020, 274, 119120.

37 P. Li, W. Fu, P. Zhuang, Y. Cao, C. Tang, A. B. Watson, P. Dong, J. Shen and M. Ye, Small, 2019, 15, 1902535.

38 E. Cao, Z. Chen, H. Wu, P. Yu, Y. Wang, F. Xiao, S. Chen, S. Du, Y. Xie, Y. Wu and Z. Ren, Angew. Chem., Int. Ed., 2020, 59, 4154-4160. 